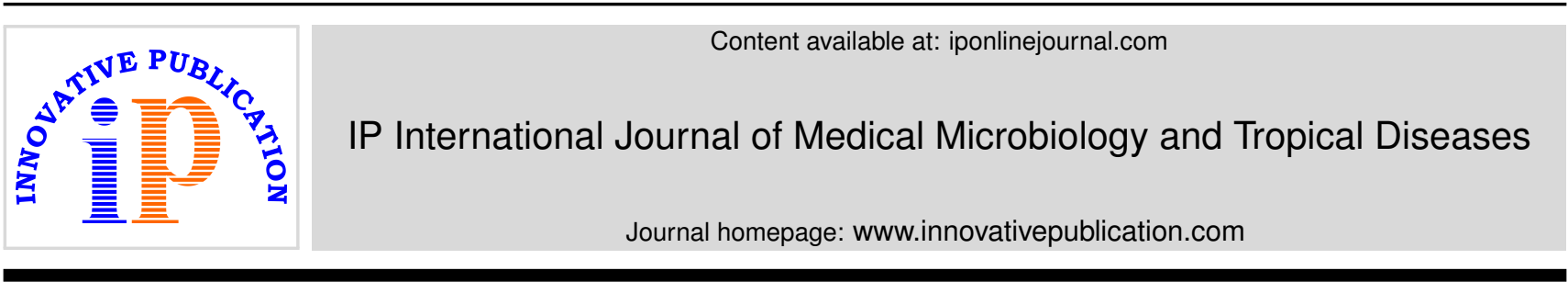

Original Research Article

\title{
Detection of Inducible clindamycin resistance in nasal carriers of Staphylococcus aureus among healthcare workers
}

\author{
Jenia Bidani ${ }^{1}$, Loveena Oberoi ${ }^{1, *}$, Anuradha Malhotra ${ }^{1}$, Shailpreet Kaur Sidhu ${ }^{1}$, \\ Sapna Soneja ${ }^{1}$ \\ ${ }^{1}$ Dept. of Microbiology, Government Medical College, Amtitsar, Punjab, India
}

\section{A R T I C L E I N F O}

\section{Article history:}

Received 17-04-2020

Accepted 18-05-2020

Available online 06-07-2020

Keywords:

S. aureus nasal carriage

Healthcare workers

MRSA

Inducible Clindamycin Resistance

\begin{abstract}
A B S T R A C T
Introduction : Staphylococcus aureus (S. aureus) is recognized as one of the most common microrganisms causing nosocomial and community-acquired infections. Nasal carriage of Staphylococcus aureus is becoming an increasing problem among healthcare workers and in the healthy community individuals. General populations with persistent Staphylococcus aureus nasal carriage rates at $10 \%$ to $20 \%$, and up to $50 \%$ are intermittent carriers. Furthermore, carrier levels of $25 \%$ have been reported among healthcare workers(HCW's). Strains with inducible clindamycin resistance (ICR) are difficult to detect in the routine laboratory as they appear to be resistant to erythromycin and sensitive to clindamycin in vitro when not placed adjacent to each other. In such cases, in vivo treatment with clindamycin may select constitutive erm mutants leading to clinical therapeutic failure.

Materials and Methods: 100 nasal swabs samples were collected during January 2019 to November 2019. Isolates were identified using standard microbioloical procedures and MRSA determined by the disk diffusion method. The D-test was performed for detection of Inducible clindamycin resistance isolates with Clinical Laboratory Standard Institute guidelines.

Results: Out of 18 S. aureus isolated, 33.3 were MRSA. Of 18 S. aureus isolates tested for ICR by D-test, 6 $(33.3 \%)$ yielded inducible resistance. Inducible clindamycin resistance was found to be significantly higher among MRSA than MSSA isolates.

Conclusion: Beside personal hygiene practices of healthcare workers, regular implementation of infection control practices, including screening of nasal carriages and microbial flora in our hospital are necessary to prevent spread of MRSA. It is also advisable to perform routine nasal decolonization of healthcare workers using mupirocin ointment to prevent transmission of these organisms.

(C) 2020 Published by Innovative Publication. This is an open access article under the CC BY-NC license (https://creativecommons.org/licenses/by-nc/4.0/)
\end{abstract}

\section{Introduction}

Staphylococcus aureus (S. aureus) is one of the most common bacteria causing nosocomial infection. ${ }^{1}$ Although Staphylococcus species is an asymptomatic colonizer but may causes skin and soft tissue infections, wound infections, ventilator-associated pneumonias, communityacquired pneumonia, necrotizing pneumonia, necrotizing fasciitis, and sepsis. ${ }^{2}$

Nasal carriage of Staphylococcus aureus among heathcare workers is becoming an alarming problem. In hospital

\footnotetext{
* Corresponding author.

E-mail address: jiyarock35@gmail.com (L. Oberoi).
}

environment, healthcare workers and asymptomatically colonized patients are the major source of MRSA. Carrier levels of $25 \%$ have been reported among healthcare workers(HCW's). ${ }^{3}$

The increasing prevalence of methicillin resistance among Staphylococci is an emerging problem not because of resistance to methicillin but also resistance to macrolide-lincosamide-streptogramin $\mathrm{B}\left(\mathrm{MLS}_{B}\right)$ antibiotics. Clindamycin is preferred agent of $\mathrm{MLS}_{B}$ but due to its extensive use, number of Staphylococcal strains resistance to this drug have been isolated. ${ }^{4,5}$ Due to modification of target site mediated by erm genes; Constitutive or Inducible 
Resistance to Clindamycin in presence of Erythromycin have been found. ${ }^{5,6}$ Clindamycin resistance among $S$. aureus isolates appears to be susceptible to clindamycin in the absence of erythromycin disk or when not placed at a recommended distance during routine antimicrobial susceptibility testing. In vivo treatment of such cases with clindamycin drug may select constitutive erm mutants resulting in no cure.

\section{Materials and Methods}

A cross-sectional prospective study was carried out in the Department of Microbiology, Government Medical College, Amritsar, Punjab for 11 months from January 2019 to November 2019. A total of 100 nursing staff, doctors and residents participated in the study. Nasal samples were collected by rotating sterile cotton swabs inside anterior nares of HCW's. These nasal swabs were inoculated on Mannitol salt agar and blood agar and incubated at $37^{\circ} \mathrm{C}$ for $24 \mathrm{~h}$. S. aureus was identified by standard microbiological procedures. ${ }^{7}$ Antimicrobial susceptibility testing was performed by the Kirby-Bauer disk diffusion method and interpreted as per the CLSI recommendations. ${ }^{8}$ The Antibiotics used in the study were Ampicillin $(10 \mu \mathrm{g})$, Gentamycin $(10 \mu \mathrm{g}), \operatorname{Amikacin}(30 \mu \mathrm{g})$, Ciprofloxacin $(5 \mu \mathrm{g})$, Teicoplanin $(30 \mu \mathrm{g})$, Linezolid( $30 \mu \mathrm{g})$. All cultured plates were aerobically incubated at $37^{\circ} \mathrm{C}$ for 24 hours before the zone sizes were recorded. A disk diffusion method with cefoxitin $(30 \mu \mathrm{g})$ was used to detect Methicillin Resistant Staphylococcus Aureus strains. ${ }^{9}$ MRSA ATCC 43300 and MSSA ATCC 25923 were used as a control strains. ${ }^{8}$ Inducible clindamycin resitance was detected by D-test. Quality control was performed by $S$. aureus ATCC 25923. ${ }^{10}$

\section{Results}

Out of the 100 nasal swab samples examined, 46 were culture positive. Out of the 46 culture positive, 18 were found to be $S$. aureus.

$18 \mathrm{~S}$. aureus isolates were screened for the presence of Methicillin Resistance; $33.3 \%$ were found to be MRSA and $66.6 \%$ were MSSA as shown in Fig 1.

The results of antimicrobial susceptibility test of the $S$. aureus isolated from healthcare workers are given in Figure 2.

Both MRSA and MSSA showed maximum resistance to Ampicillin, Erythromycin Ciprofloxacin and Clindamycin. All the isolates show 100\% susceptibility to Teicoplanin and Vancomycin.

Eighteen $S$. aureus isolates (6 MRSA and 12 MSSA) tested for ICR by D-test, 6 (33.33\%) yielded inducible resistance. Inducible clindamycin resistance was found to be notably higher among MRSA than MSSA isolates.

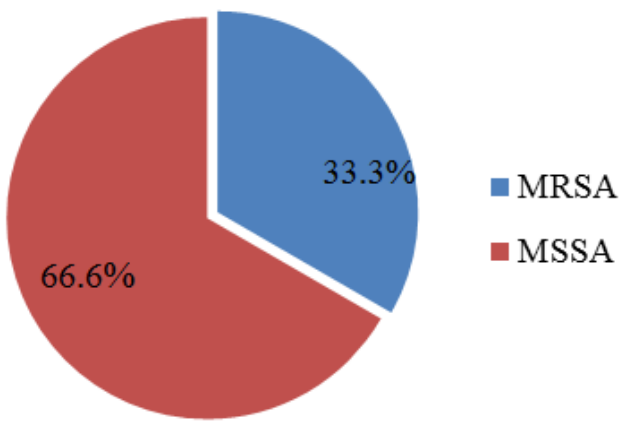

Fig. 1:

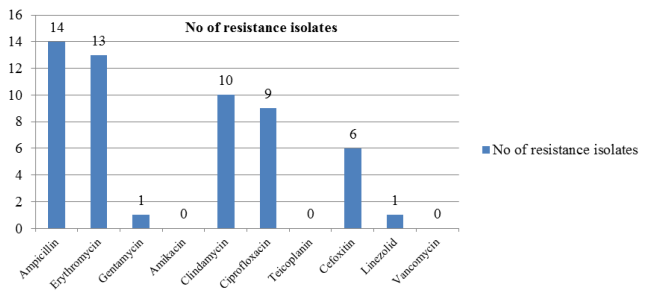

Fig. 2:

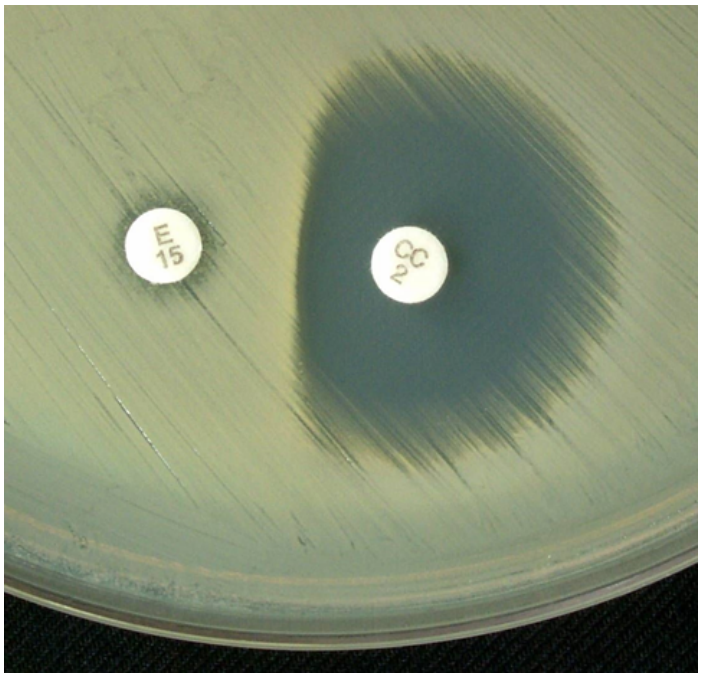

Fig. 3:

Table 1:

\begin{tabular}{lll}
\hline Type of isolate & No of isolates & $\begin{array}{l}\text { No. of inducible } \\
\text { clindamycin resistance }\end{array}$ \\
MRSA & 6 & 4 \\
MSSA & 12 & 2 \\
TOTAL & 18 & 6 \\
\hline
\end{tabular}




\section{Discussion}

Determination of colonization prevalence provides a useful estimate of the potential for development of $S$. aureus infections. ${ }^{11,12}$ In the present study, the prevalence of $S$. aureus nasal colonization among healthcare workers was $18 \%$ which is similar to the findings by Radhakrishna $\mathrm{M}$ et al $(17.5 \%) .{ }^{13}$ These findings are also similar to study carried out in tertiary care hospital in Assam where prevalence was found to be $22.22 \% .{ }^{14}$ However, study conducted in Gauhati Medical College, and in Burdwan Medical College nasal colonization was found to be $36 \%$ and $34.80 \%$ respectively. ${ }^{15,16}$

Surveillance of HCW's for MRSA carriage is essential specially in critical care areas as they pose a threat to infection control practices. ${ }^{17}$ Different studies have described a higher prevalence of MRSA colonization among persons of low socio-economic status in the general community, may be associated with crowding, limited access to healthcare, or barriers to maintaining adequate hygiene. ${ }^{18}$ The carriage rate of MRSA in present study was 6\% which is similar to study carried out in Delhi by Goyal $\mathrm{R}$ et al (6.6\%). Another study carried out in Rajajinagar, Banglore by Malini J et al, MRSA carriage rate was found to be $8 \% .{ }^{19}$ A study from Nigeria by Fadeyi A et al reported $38.9 \%$ carriage rate. ${ }^{20}$

The overall prevalence ICR was $33.3 \%$; with $66.6 \%$ of MRSA and $16.6 \%$ of MSSA. Similar study by Mahmoud AM et al found overall prevalence of ICR 29.4; with 43.5\% of MRSA and $20.9 \%$ of MSSA. ${ }^{21}$ In contrast, Patel et al found the overall prevalence was $52 \%$, with $50 \%$ of MRSA and $60 \%$ of MSSA isolates exhibiting ICR. ${ }^{4}$ These findings with our current results indicate the significant occurrence of ICR between MRSA and MSSA. Therefore, antimicrobial susceptibility data of ICR isolates should be evaluated routinely in each infection caused by $S$. aureus before starting the treatment.

\section{Conclusion}

Increasing incidence of MRSA and Inducible Clindamycin resistance among HCW's is a cause of concern leaving few therapeutic options. Regular implementation of infection control practices, including screening of nasal carriages are necessary to prevent spread of MRSA. It is also advisable to perform routine nasal decolonization of healthcare workers using mupirocin ointment to prevent transmission of these organisms.

\section{Source of Funding}

None.

\section{Conflict of Interest}

None.

\section{References}

1. Singh S, Malhotra R, Grover P, Bansal R, Galhotra S, Kaur $\mathrm{R}$, et al. Antimicrobial resistance profile of Methicillin-resistant Staphylococcus aureus colonizing the anterior nares of health-care workers and outpatients attending the remotely located tertiary care hospital of North India. J Lab Physicians. 2017;9(04):317-21.

2. Basak S, Mallick SK, Bose S. Methicillin resistant Staphylococcus aureus (MRSA) infections. J Clin Diagn Res. 2010;4:2111-5.

3. Naber CK. Staphylococcus aureusBacteremia: Epidemiology, Pathophysiology, and Management Strategies. Clin Infect Dis. 2009;48(s4):S231-37.

4. Deotale V, Mendiratta DK, Raut U, Narang P. Inducible clindamycin resistance inStaphylococcus aureusisolated from clinical samples. Indian J Med Microbiol. 2010;28(2):124-6.

5. Gupta V, Datta P, Rani H, Chander J. Inducible clindamycin resistance in Staphylococcus aureus: A study from North India. J Postgrad Med. 2009;55(3):176-9.

6. Patel M, Waites KB, Moser SA, Cloud GA, Hoesley CJ. Prevalence of Inducible Clindamycin Resistance among Community- and HospitalAssociated Staphylococcus aureus Isolates. J Clin Microbiol. 2006;44(7):2481-4.

7. Collee JG, Milrs RS, Watt B. Tests for identification of bacteria. In: JG C, AG F, BP M, A S, editors. Mackie \& McCartney Practical Medical Microbiology . New Delhi: Elsevier India pvt. Ltd; 2006. p. 796-800.

8. CLSI. Performance Standards for Antimicrobial Disk Susceptibility Tests. 13th ed. CLSI standard M02. Wayne, PA; Clinical and Laboratory Standards Institute; 2018.

9. Landuyt HV, Cauwelier B, Gordts B, Descheemaecker P. Evaluation of a disk diffusion method with cefoxitin (30 microg) for detection of methicillin-resistant Staphylococcus aureus. Eur J Clin Microbiol Infect Dis. 2004;23(5):389-92.

10. Fiebelkorn KR, Crawford SA, McElmeel ML, Jorgensen JH. Practical Disk Diffusion Method for Detection of Inducible Clindamycin Resistance in Staphylococcus aureus and CoagulaseNegative Staphylococci. J Clin Microbiol. 2003;41(10):4740-4.

11. Yazgi H, Ertek M, Ozbek A, Kadanali A. Nasal carriage of Staphylococcus aureus in hospital personnel and the normal population and antibiotic resistance of the isolates. Mikrobiyol Bul. 2003;37(2-3):137-142.

12. Kuehnert MJ, Kruszon-Moran D, Hill HA. Prevalence of Staphylococcus aureus nasal colonization in the United States. J Infect Dis. 2001;193(2):172-9.

13. Mohite RV. Study of Nasal Carriage of Staphylococcus Aureus with Special Reference to Methicillin Resistance among Nursing Staff . Arch Clin Microbiol. 2015;7.

14. Rongpharpi SR, Hazarika NK, Kalita H. The Prevalence of Nasal Carriage of Staphylococcus Aureus Among Healthcare Workers at a Tertiary Care Hospital in Assam with Special Reference to MRSA. $J$ Clin Diagn Res. 2013;7(2):257-60.

15. Mayuri G, Rasul A, Sharma A, Mohan D. Nasal and hand carriage of methicillin-resistant Staphylococcus aureus among health care workers of a tertiary care hospital in north-east India. IOSR J Dent Med Sci. 2017;16:36-41.

16. Mondal H, Gupta I, Nandi P, Ghosh P, Chattopadhyay GDS, Mitra. Nasal screening of healthcare workers for nasal carriage of methicillin resistance staphylococcus aureus, vancomycin resistance staphylococcus aureus and prevalence of nasal colonization with staphylococcus aureus in burdwan medical college and hospital. Int J Contemp Med Res. 2016;3(11):3342-6.

17. Ahmed AO, van Belkum A, Fahal AH, Elnor AEA, Abougroun ESAM, Bergh MFQV, et al. Nasal Carriage of Staphylococcus aureusand Epidemiology of Surgical-Site Infections in a Sudanese University Hospital. J Clin Microbiol. 1998;36(12):3614-8.

18. Gorwitz RJ, Kruszon-Moran D, Mcallister SK. Changes in the prevalence of nasal colonization with Staphylococcus aureus in the United States. J Infect Dis. 2001;197(9):1226-34. 
19. Hema N, Raj N, Chaithanya ED, Chincholi R, Iswariya M, Hema KN. Prevalence of nasal carriers of methicillin-resistant Staphylococcus aureus among dental students: An in vivo study. J Oral Maxillofac Pathol. 2017;21(3):356-9.

20. Abayomi F, Benjamin B, Olanrewaju O, Omotayo A, N AMA, Timothy O, et al. Methicilin Resistant Staphylococcus aureus Carriage amongst Healthcare Workers of the Critical Care Units in a Nigerian Hospital. Am J Inf Dis. 2010;6.

21. Mahmoud AM, Albadawy HS, Bolis SM, Bilal NE, Ahmed AO, Ibrahim ME. Inducible clindamycin resistance and nasal carriage rates of $<\mathrm{i}>$ Staphylococcus aureus $</ \mathrm{i}>$ among healthcare workers and community members. Afr Health Sci. 2015;15(3):861-7.

\section{Author biography}

Jenia Bidani Junior Resident

\section{Loveena Oberoi HOD}

Anuradha Malhotra Associate Professor

Shailpreet Kaur Sidhu Associate Professor

Sapna Soneja Associate Professor

Cite this article: Bidani J, Oberoi L, Malhotra A, Sidhu SK, Soneja S. Detection of Inducible clindamycin resistance in nasal carriers of Staphylococcus aureus among healthcare workers. IP Int J Med Microbiol Trop Dis 2020;5(2):103-106. 